\title{
Clinical Analysis on the Treatment of Early Diabetic Nephropathy with Gushen Huoxue Method
}

\author{
Xuan Zhang', Feng Li ${ }^{2, *}$ \\ ${ }^{1}$ Shaanxi University of Traditional Chinese Medicine, Xianyang, 714000, China. \\ ${ }^{2}$ Internal Medicine Center of TCM of PLA, Xijing Hospital of Air Force Military Medical University, Xi'an, 710032, China.
}

\begin{abstract}
How to cite this paper: Xuan Zhang, Feng Li. (2021) Clinical Analysis on the Treatment of Early Diabetic Nephropathy with Gushen Huoxue Method. International Journal of Clinical and Experimental Medicine Research, 5(1), 70-74.

DOI: 10.26855/ijcemr.2021.01.009
\end{abstract}

Received: December 21, 2020

Accepted: January 15, 2021

Published: January 29, 2021

*Corresponding author: Feng $\mathrm{Li}$, Internal Medicine Center of TCM of PLA, Xijing Hospital of Air Force Military Medical University, Xi'an, 710032, China.

\begin{abstract}
Objective: To explore the curative effect of Gushen Huoxue Method in the treatment of early diabetic nephropathy. Methods: 100 patients with early diabetic nephropathy in our hospital from January 2019 to January 2020 were randomly selected and randomly divided into study group and control group, 50 cases in each group. The patients in the study group were treated with Gushen Huoxue Decoction on the basis of conventional treatment, while the control group was treated with conventional treatment. The fasting blood glucose and $2 \mathrm{~h}$ postprandial blood glucose levels of the two groups were compared and analyzed. Results: After two courses of treatment, the indexes of fasting blood glucose and postprandial $2 \mathrm{~h}$ blood glucose in the study group were significantly better than those in the control group, and the difference between the two groups was statistically significant $(P<0.05)$. After treatment, TC, TG, HDL-C and LDL-C in the treatment group were significantly different from those in the control group $(P<0.05)$, and the treatment group was better than the control group. After treatment, SCR, bun, mAlb, EGFR and Cys-C in the treatment group were significantly different from those in the control group $(P<0.05)$, and the treatment group was better than the control group. The total effective rate of the study group was higher than that of the control group, the difference was statistically significant $(P<0.05)$. Conclusion: The method of invigorating the kidney and activating blood circulation has obvious curative effect in the treatment of patients with early diabetic nephropathy.
\end{abstract}

\section{Keywords}

Bushen Huoxue Method, Diabetic nephropathy, Gliquidone

Diabetic nephropathy is the main microvascular complication of diabetes mellitus. The main pathological changes are glomerulosclerosis between capillaries. Diabetic nephropathy can be divided into five stages. Once in stage IV diabetic nephropathy, renal function will be irreversible damage, so the treatment of early stage III diabetic nephropathy is very important. The treatment of early diabetic nephropathy in modern medicine is mainly to control blood glucose, which can be combined with angiotensin-converting enzyme inhibitor (ACEI), but it cannot achieve satisfactory clinical effect. Diabetic nephropathy is one of the more common and serious complications in diabetes. In the process of its occurrence, some diabetic patients will always experience normal proteinuria, microalbuminuria and clinical albuminuria in the process of disease development. The renal function of diabetic patients will be 
constantly damaged, and even gradually evolve into diabetic nephropathy Kidney disease is more serious in the case of diabetic patients will appear the phenomenon of renal failure, and ultimately lead to the death of patients with diabetes. In the treatment of diabetic nephropathy, the conventional treatment is mainly to take western medicine treatment, which can effectively control the biochemical indicators of patients, but this treatment cannot effectively repair the renal function of patients [1]. Conventional western medicine combined with kidney strengthening and blood activating therapy is used in the treatment of patients with early diabetic nephropathy In order to be able to more clearly and specifically observe the specific therapeutic effect of Gushen Huoxue Method on patients with diabetic nephropathy, the author conducts a specific study on the treatment process and effect of patients with early diabetic nephropathy treated in our hospital, and compares and analyzes the specific results of different treatment methods, which are reported as follows.

\section{Materials and methods}

\subsection{General information}

100 patients with early diabetic nephropathy in our hospital from January 2019 to January 2020 were randomly selected and randomly divided into the study group and the control group, 50 cases in each group. In the study group, 33 cases were male and 27 cases were female, with an average age of $(45.5 \pm 11.4)$ years old, with a course of 6-10 months and an average duration of $(7.31 \pm 1.02)$ months. In the control group, there were 29 males and 31 females, aged 33-69 The average age was $(47.1 \pm 13.6)$ years old. The course of disease was 6-11 months and the average course was $(7.82 \pm 0.91)$ months. There was no significant difference in gender, age and course of disease between the two groups $(P>0.05)$.

\subsection{Diagnosis, inclusion and exclusion criteria}

Diagnostic criteria: Western medicine in the diagnosis of diabetic nephropathy patients, mainly to the specific diagnostic criteria of diabetes, traditional Chinese medicine in the process of diagnosis of diabetic nephropathy, mainly to diabetes diagnostic criteria as the main reference standard, early diabetic nephropathy patients mainly manifested as dry mouth and thirst and physical fatigue and other aspects of symptoms [2]. The inclusion criteria were formulated according to the Chinese guidelines for the prevention and treatment of type 2 diabetes mellitus (2013 Edition). There was a clear history of diabetes mellitus, early DN was DN stage III: UAER was more than 20 $\mu \mathrm{g} / \mathrm{min}$ or 30-300 mg / $24 \mathrm{~h}$ in 6 months. Exclusion criteria: (1) patients with type I diabetes mellitus; (2) patients with secondary renal damage caused by hypertension; (3) patients with diabetes complicated with acute complications; (4) those with physical and mental state (infants, minors, elderly people, mental illness, malignant tumor, heart failure, etc.) who are not suitable to participate in this study; (5) pregnant or lactating women and women with pregnancy plan in the near future; and (6) the same period The patients who were treated with other traditional Chinese medicine and affected the judgment of curative effect.

\subsection{Therapeutic method}

\subsubsection{Control group}

Exercise and diet management and scientific collocation, and conventional diabetes treatment, oral hypoglycemic drug Gliquidone (Tianjin Jinshi Pharmaceutical Co., Ltd., Guoyao Zhunzi h20084004), and insulin [Hong Kong Fengshun International Pharmaceutical Co., Ltd., Yu Wei Shi Zi (2008) No. 0050] Benazepril hydrochloride tablets (Shanghai Xinya pharmaceutical Minhang Co., Ltd., Guoyao Zhunzi h20044840) 10 mg, once a day, were used for blood pressure control. Drugs and food that have effects on the kidney are prohibited. Two courses of treatment.

\subsubsection{Research group}

On the basis of the control group, the combination of Gushen huoxue prescription, the specific components of Gushen Huoxue Prescription include $10 \mathrm{~g}$ of Radix Polygoni Multiflori, $30 \mathrm{~g}$ of Astragalus membranaceus and Salvia miltiorrhiza, $10 \mathrm{~g}$ of prepared radix rehmanniae, mulberry, Ligustrum Lucidi, Fructus Psoraleae, polygonatum, Radix et Rhizoma Rhei and semen cuscutae, 1 dose per day, Decoction in water, 1 time in the morning and 1 in the evening, 2 courses of treatment.

\subsection{Observation items}

(1) Blood sugar. After treatment, the fasting blood glucose, $2 \mathrm{~h}$ postprandial blood glucose and glycosylated hemoglobin of the two groups were observed and compared; (2) blood lipid. It includes cholesterol (TC), triglyceride (TG), high density lipoprotein cholesterol (HDL-C) and low-density lipoprotein cholesterol (LDL-C); (3) renal 
function. Serum creatinine (SCR), blood urea nitrogen (BUN), urinary microalbumin (mAlb), glomerular filtration rate (EGFR) and cystatin C (Cys-C) were measured.

\subsection{Judgment of therapeutic effect}

Significant effect, the patient's symptoms gradually disappeared, blood sugar decreased or returned to normal; effective, clinical symptoms improved, blood sugar decreased significantly; ineffective, compared with before treatment, symptoms did not significantly improve or aggravate [3]..

\subsection{Statistical methods}

SPSS 19.0 software was used to process the data. The measurement data was represented by $(\bar{x} \pm s)$ and the count data was represented by percentage. The chi square test was used. The difference was statistically significant $(P<0.05)$.

\section{Results}

\subsection{Comparison of blood glucose levels between the two groups}

The blood glucose control of the two groups was compared, the blood glucose control of the study group was better than that of the control group, the difference was statistically significant $(P<0.05)$, see Table 1 .

Table 1. Comparison of blood glucose levels between the two groups $(\bar{x} \pm s)$

\begin{tabular}{ccccc}
\hline Groups & Time & FBG $(\mathrm{mmol} / \mathrm{L})$ & 2hPG $(\mathrm{mmol} / \mathrm{L})$ & HbA1c $(\%)$ \\
\hline Research group $(\mathrm{n}=50)$ & Before treatment & $8.1 \pm 0.8$ & $12.4 \pm 1.4$ & $8.5 \pm 0.7$ \\
& After treatment & $6.9 \pm 0.4$ & $8.8 \pm 1.3$ & $7.0 \pm 0.4$ \\
Control group $(\mathrm{n}=50)$ & Before treatment & $8.2 \pm 0.5$ & $12.9 \pm 1.3$ & $8.3 \pm 0.7$ \\
& After treatment & $7.4 \pm 0.3$ & $9.0 \pm 1.2$ & $7.6 \pm 0.3$ \\
\hline
\end{tabular}

\subsection{Comparison of blood lipid levels between the two groups}

After treatment, TC, TG, HDL-C and LDL-C in the treatment group were compared with those in the control group, and the differences were statistically significant $(P<0.05)$. The treatment group was better than the control group, as shown in Table 2.

Table 2. Comparison of blood lipid levels between the two groups $(\mathrm{mmol} / \mathrm{L}, \bar{x} \pm s)$

\begin{tabular}{cccccc}
\hline Groups & Time & TC & TG & HDL-C & LDL-C \\
\hline Research group $(\mathrm{n}=50)$ & Before treatment & $6.8 \pm 0.5$ & $3.5 \pm 0.4$ & $0.55 \pm 0.05$ & $4.6 \pm 0.4$ \\
& After treatment & $4.4 \pm 0.7$ & $1.9 \pm 0.4$ & $1.43 \pm 0.40$ & $2.4 \pm 0.5$ \\
\multirow{2}{*}{ Control group $(\mathrm{n}=50)$} & Before treatment & $6.8 \pm 0.7$ & $3.6 \pm 0.5$ & $0.53 \pm 0.04$ & $4.4 \pm 0.6$ \\
& After treatment & $5.8 \pm 1.1$ & $2.5 \pm 0.5$ & $0.97 \pm 0.30$ & $3.2 \pm 0.6$ \\
\hline
\end{tabular}

\subsection{Comparison of renal function indexes between the two groups}

After treatment, SCR, bun, mAlb, EGFR and Cys-C in the treatment group were compared with those in the control group, and the differences were statistically significant $(P<0.05)$. The treatment group was better than the control group, as shown in Table 3.

Table 3. Comparison of renal function indexes between the two groups $(\bar{x} \pm s)$

\begin{tabular}{ccccccc}
\hline Groups & Time & $\begin{array}{c}\mathrm{SCr} \\
(\mu \mathrm{mol} / \mathrm{L})\end{array}$ & $\begin{array}{c}\mathrm{BUN} \\
(\mathrm{mmol} / \mathrm{L})\end{array}$ & $\begin{array}{c}\mathrm{mAlb} \\
(\mathrm{mg} / \mathrm{gG})\end{array}$ & $\begin{array}{c}\text { eGFR } \\
(\mathrm{ml} / \mathrm{min})\end{array}$ & $\begin{array}{c}\text { Cys-C } \\
(\mathrm{mg} / \mathrm{L})\end{array}$ \\
\hline Research group $(\mathrm{n}=50)$ & Before treatment & $94.7 \pm 1.7$ & $7.9 \pm 0.8$ & $51.7 \pm 5.1$ & $66.1 \pm 3.6$ & $2.4 \pm 0.6$ \\
& After treatment & $79.6 \pm 2.3$ & $5.0 \pm 0.4$ & $48.3 \pm 4.0$ & $74.7 \pm 5.1$ & $1.4 \pm 0.3$ \\
Control group (n=50) & Before treatment & $95.2 \pm 2.1$ & $7.9 \pm 0.8$ & $54.7 \pm 7.6$ & $65.2 \pm 6.7$ & $2.3 \pm 0.5$ \\
& After treatment & $84.0 \pm 3.2$ & $5.5 \pm 0.4$ & $52.3 \pm 2.9$ & $67.2 \pm 7.0$ & $1.9 \pm 0.4$ \\
\hline
\end{tabular}




\subsection{Comparison of curative effect between the two groups}

The total effective rate of the study group was higher than that of the control group, the difference was statistically significant $(P<0.05)$, see Table 4 .

Table 4. Comparison of curative effect between the two groups (n/\%)

\begin{tabular}{ccccc}
\hline Groups & Markedly effective & Effective & Invalid & Total effective \\
\hline Research group $(\mathrm{n}=50)$ & $13(26.0)$ & $25(50.0)$ & $12(24.0)$ & $38(76.0)$ \\
Control group $(\mathrm{n}=50)$ & $9(18.0)$ & $13(26.0)$ & $28(56.0)$ & $22(44.0)$ \\
$\chi^{2}$ & & & 16.010 \\
$P$ & & & $<0.05$ \\
\hline
\end{tabular}

\section{Discussion}

The exact pathogenesis of DN has not been elucidated yet. It may include genetic, glucose and lipid metabolism disorders, oxidative stress, hemodynamic changes, vasoactive substances and other factors. Early diabetic nephropathy stage, also known as continuous microalbuminuria stage, EGFR Renal pathology showed glomerular nodular lesions and arteriolar hyalinization, and microalbuminuria. If at this time, through drug intervention to reduce microalbuminuria excretion, can delay the progression of kidney disease. Once patients appear persistent large amount of proteinuria, the condition is often irreversible, until end-stage renal failure.

With the development of society, people's living standard and the pace of life are accelerating. People are having more unhealthy habits and irregular diet during their lives. According to the specific clinical analysis, the incidence rate of diabetes is increasing year by year [4]. At the same time, when the urinary tract infection is excluded, diabetic patients are prone to microalbuminuria or massive proteinuria. This condition can be diagnosed as diabetic nephropathy. Diabetic nephropathy (DN) is one of the main microvascular complications of diabetes, and also the main cause of end-stage renal disease (ESRD). The main clinical manifestations of DN are glomerular injury and secondary proteinuria. Early DN renal injury is reversible, and once it enters dominant proteinuria, the renal damage is irreversible [5]. At present, western medicine mainly adopts the methods of controlling blood glucose, blood pressure and regulating blood lipid to delay the progress of DN, but it cannot reverse the pathological process. Diabetic nephropathy is the main microvascular complication of diabetes mellitus. The main pathological changes are glomerulosclerosis between capillaries. Diabetic nephropathy can be divided into five stages. Once in stage IV diabetic nephropathy, renal function will be irreversible damage, so the treatment of early stage III diabetic nephropathy is very important [6]. However, the effect of ACEI on diabetic nephropathy cannot be controlled by angiotensin converting enzyme [7].

In TCM theory, DN belongs to diabetes, turbid urine, edema, fatigue and other diseases. In traditional Chinese medicine, it can be considered that the kidney of diabetic patients is damaged to a certain extent due to the long-term injury of yin and gas consumption, Qi and yin deficiency and Yin damage, which causes the formation of diabetic nephropathy. In the process of specific research, Qi and yin deficiency is also an important cause of diabetes. This research method is mainly made of Astragalus membranaceus or Salvia miltiorrhiza. Astragalus membranaceus has the effect of tonifying the kidney and Qi and promoting blood circulation. This medicinal material can effectively improve the microcirculation of patients, reduce the excretion of urine protein and protect the kidney. Salvia miltiorrhiza also has the function of regulating renal function, which can effectively reduce the occurrence of proteinuria in patients with diabetic nephropathy. The combination of these herbs in the specific treatment prescription can play a role in strengthening the kidney and promoting blood circulation in the treatment of patients with diabetic nephropathy [8].

In order to observe the clinical efficacy of Yiqi Huoxue Gushen method in the treatment of early diabetic nephropathy, 150 cases of early diabetic nephropathy were randomly divided into two groups, 76 cases in the treatment group and 74 cases in the control group. The control group was given routine lifestyle guidance, hypoglycemic, antihypertensive and lipid-lowering treatment, while the treatment group was given Yiqi Huoxue Gushen Decoction on the basis of the control group. The course of treatment was 3 months in both groups. Fasting blood glucose, glycosylated hemoglobin, blood lipid, renal function and clinical efficacy were observed. The results showed that the total effective rate was $81.6 \%$ in the treatment group and $67.6 \%$ in the control group. After treatment, fasting blood glucose (FBG), glycosylated hemoglobin (HbA1c), cholesterol (TC), triglyceride (TG), high-density lipoprotein cholesterol (HDL-C), low-density lipoprotein cholesterol (LDL-C), serum creatinine (SCR), blood urea 
nitrogen (BUN), microalbuminuria (mAlb), glomerular filtration rate (EGFR), Cystatin C (Cys-C) were measured in the treatment group Compared with the control group, the difference was statistically significant $(P<0.05)$, and the treatment group was better than the control group. It is concluded that Yiqi Huoxue Gushen therapy in the treatment of early diabetic nephropathy, in addition to lowering blood glucose and lipid, can significantly improve renal function [9]. Relevant medical research also shows that early diabetic nephropathy can significantly improve the cure rate of the disease on the basis of controlling blood glucose, and the exact mechanism of action needs further study [10].

In this study, after two courses of treatment and observation, it can be found that the fasting blood glucose and 2 hours after meal of patients in the study group Blood glucose indicators were significantly better than the control group, the difference between the two groups was statistically significant. After treatment, TC, TG, HDL-C and LDL-C in the treatment group were compared with those in the control group, and the differences were statistically significant, and the treatment group was better than the control group. After treatment, SCR, bun, mAlb, EGFR and Cys-C in the treatment group were compared with those in the control group, and the differences were statistically significant, and the treatment group was better than the control group. The total effective rate of the study group was $76.67 \%$, which was significantly higher than that of the control group (43.33\%). It is suggested that this prescription can reduce the urinary protein of DN patients, reduce the renal injury, delay the progression of DN, and play a role in stabilizing the disease. At the same time, it also showed that after the treatment of diabetic nephropathy patients with Gushen Huoxue Method, the renal function indexes of patients could be improved effectively, and the blood glucose of patients could be effectively controlled.

To sum up, the effect of Gushen Huoxue recipe combined with the traditional treatment of diabetic nephropathy is obvious.

\section{Funding}

This study was approved by National Natural Science Foundation of China (81273622).

\section{References}

[1] Quan Shijian, Wu Chunying. (2018). Clinical observation of Yiqi Huoxue Gushen therapy in the treatment of early diabetic nephropathy [J]. New Chinese Medicine, 50(5): 84-86.

[2] Chen Xiaoliang. (2017). Observation on the therapeutic effect of Gushen Huoxue therapy on early diabetic nephropathy [J]. Shenzhen Journal of Integrated Traditional Chinese and Western medicine, 27(13): 68-70.

[3] Lai Xiaolin, Guo Lihua, Liu Changzhi, et al. (2018). Clinical study of Jianpi Gushen Huoxue Decoction Combined with irbesartan in the treatment of early diabetic nephropathy [J]. Basic Medicine Forum, 22(5): 579-580.

[4] Luo Zhaoqiang, Zhou Shixiang. (2019). Effect analysis of Jianpi Gushen Huoxue Decoction Combined with irbesartan in the treatment of early diabetic nephropathy [J]. Medical frontier, 9(24): 121-122.

[5] Yin Di, Zhang Xu, Cao Juan, et al. (2018). Clinical effect analysis of Jianpi Gushen Huoxue Decoction Combined with irbesartan in the treatment of early diabetic nephropathy [J]. Diabetes New World, 21(16): 189-191194.

[6] Zhang Like. (2018). Analysis on the effect of Jianpi Gushen Huoxue Decoction Combined with irbesartan in the treatment of early diabetic nephropathy and its impact on renal function [J]. Medical Frontier, 8(28): 314-315.

[7] Tang Xiaomei, Zu Yizhi, Chen Qiu. (2016). Learning from Professor Zhang Farong on the treatment of proteinuria in diabetic nephropathy [J]. Medical Information, 29(15): 284-284.

[8] Gao Huijuan, Feng Xingzhong. (2019). Analysis on the treatment of diabetic nephropathy with Jianpi Gushen, Huatan Huoxue Jiedu method [J]. Chinese Journal of traditional Chinese Medicine, 34(1): 155-158.

[9] Wang Huiling, Qin Gangxin, Zhao Yage, et al. (2018). Effect of Jianpi Gushen Huoxue Decoction Combined with irbesartan on serum sICAM-1, MCP-1 and ET-1 levels in patients with diabetic nephropathy [J]. Sichuan Traditional Chinese Medicine, 36(12): 99-102.

[10] Lu Shuquan, Su xiuhai. (2020). Experience of chief physician Su xiuhai in diagnosis and treatment of clinical diabetic nephropathy [J]. Journal of Modern Integrated Chinese and Western Medicine, 29(30): 3354-3357. 